\title{
Personalization of Political Discoures On Social Media
}

\author{
Cristina Florentina Moise, University of Bucharest, cristi- \\ na.florentina.moise@gmail.com
}

\begin{abstract}
According to Phillips and Young, success in politics is now highly influenced by the online activities of political institutions. Within these platforms, politicians can exchange views on the latest partisan developments or hot topics, inviting the public and citizens to comment, share ideas and adhere to their political programs [Phililips and Young et al 2009].
\end{abstract}

According to Sundar, Kalyanaraman, and Brown (2003) interactivity is usually associated with positive perception. The user will only make an effort to search and read the information if he feels engaged with the political party or message [Sundar, Kalyanaraman and Brown et al 2003]. Online political engagement is largely restricted to people already active in politics and on the Internet. Other audiences are less responsive [Tenhunen and Karvelyte et al 2015].

Nevertheless, in the last years, social media has reshaped its structures and methods of contemporary political communication. The public became more active and willing to react to political message even though it is not partisan of a political party.

That is why, in the last years, politicians have a significant interest to have a two-way communication with their citizens, to discover their opinions and feelings about different ideas. Therefore, it is essential to allocate resources for sentiment analysis, which is also called opinion mining (one of the most active research areas in natural language processing since early 2000 [Liu et al 2012]).

\section{Introduction}

Online environment especially, social media channels are the most used tools for public communication within societies. In particular, for having a direct dialog with their citizens, politicians are continuously delivering message on microblogs (like Twitter) or on social networks (like Facebook) [Chilton and Schäffner et al 2002].

For political communication, it is essential to come with personalized discourses for each segment of voters, to get the insights quickly and to have the flexibility to adapt the message according to the context and to the people reaction.

This paper overviews the Majoritas ecosystem (https://majoritas.com/), providing a complete overview of political campaigns assessment aimed to assist politicians and their staff in delivering consistent and personalized message.

The system has been running for five years being adopted on almost all continents: Brazil, the US, France, Congo, Indonesia, Moldavia, Albania, Serbia, etc.

The ecosystem contains a suite of services and applications that can outline and summarize data provided by online, offline and social media sources, assists in creating the right message to the right audience, assessing voters' behavior during the entire time of the campaign.

The solution was built to help politicians to have an iterative approach in their campaigns. It is important to adopt an 'agile' methodology when political communication strategy is defined. After each message, post or tweet, politicians need to assess the impact. What is the reaction of their followers or, is there any response from the competition?

Majoritas Social Media Room (one of the application of Majoritas ecosystem) was designed as a cloud political communication platform that assists politicians in empowering their social media presence. In other words, from a single place, the candidate can communicate diverse types of mes- 
sage within his social media community using multiple Twitter accounts and Facebook pages.

Besides delivering messages, Social Media platform was designed to get real time voters' reaction regarding candidate's posts and tweets, providing insights concerning their engagement to different topics, ideas or beliefs.

\section{Related Work}

Mullen and Malouf et al 2006, based on the posts collected from several political blogs, tried to classify if posters' orientation was either left or right. They applied the Naïve Bayes text classifier and conclude that for political sentiment analysis, the traditional word-based text classification is not adequate.

According to Yu and Hatzivassiloglou et al 2003, considering the character limitation of tweets, classifying the sentiment analysis of Twitter messages is similar to the analysis of sentence-level sentiment. It is important to consider the informal tone as well and the specialized language used on social media.

Besides sentiment analysis algorithms, TF-IDF (Term Frequency-Inverse Document Frequency) technique is also very useful to performing microblogs text mining.

Even though both: sentiment analysis and TF-IDF are considered text classification techniques, their scope is different. On the one hand, the aim of sentiment analysis is to classify texts into opinions: 'negative' or 'positive'. On the other hand, the scope of TF-IDF is to classify categories within categories.

TF-IDF calculates a weight which represents the importance of a term inside a text:

$\mathrm{TF}(\mathrm{t})=($ Number of times term $\mathrm{t}$ appears in a document) / (Total number of terms in the document) $\operatorname{IDF}(\mathrm{t})=\log _{-} \mathrm{e}($ Total number of documents / Number of documents with term $t$ in it).

Value $=\mathrm{TF} * \mathrm{IDF}$

Another important aspect that needs to be considered in translating microblogs messages into in- sights regarding posters' feelings, views, judgements, is the social network. As per Pozzi, Fersini, Mesina and Liu et al 2017, sentiment analysis in social networks is generally based on the assumption that the texts provided by the users are independent and identically distributed.

The principle of homophily [Lazarsfeld and Merton et al 1954], reveals that based on the 'friendship' relationships, connected users may be likelier to share similar opinions.

Popescu and Etzioni et al 2005 proposed PMI Pointwise Mutual Information statistics to improve aspect extraction on product reviews. They introduced the meronymy term which represents a part-whole relationship. In regard to politics, the topics used by candidates in their discourse are also a form of meronymy since these subjects are part of their campaigns.

\section{Data Gathering}

Using Majoritas Social Media platform, we collected all information from Facebook and Twitter accounts regarding a politician of which identity will be kept anonymized.

Both Twitter and Facebook provide APIs for data tracking. If Twitter offers Search API and Streaming API for tweets monitoring, Facebook provide Graph API for posts' tracking.

In addition, the paper's authors considered that the integration of the insights obtained from several social media channels, including micro blogs, generates a consistent image of the political message.

To assess the impact of a message, several data points should be analyzed and correlated.

Below are presented some conclusions obtained for a politician from Europe CEE (presidential elections).

Majoritas concluded that hashtags analysis needs to be performed in order to assess for how many times, politician's hashtags were mentioned on Facebook or Twitter. This indicator provides a strong insight regarding the impact of the message (post) on social media being linked with the meronymy term of Popescu and Etzioni, 2005: 


\begin{tabular}{|c|c|c|c|c|c|}
\hline Таg & 7 Created & Times Used fb & Times Jsed Tw & 7Times Used & 7 Used By \\
\hline 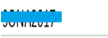 & 2/21/120177:51 & & 3442 & $24311^{\prime}$ &  \\
\hline 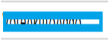 & 11/30/201615:23 & & 3412 & $11256^{\prime}$ & 14668 Our pogeses \\
\hline  & 11/23/20160:51 & & 1245 & $12441^{\prime}$ & 13568 Our pages \\
\hline tulnerat & 11/22/20161621:45 & & 55122 & $23556^{\prime}$ & 48678 Our pages \\
\hline Changelscoming & 4/24/2016 16:11 & & 24332 & $5433^{\prime}$ & 29965 \\
\hline 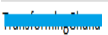 & $101 / 1201520.36$ & & 5432 & $452122^{\prime}$ & 50644 Our pages \\
\hline Changinglives & $10 / 1 / 201520: 36$ & & 6453 & $21233^{\prime}$ & 27686 Our poges \\
\hline
\end{tabular}

Table 1: Hastags Analysis

Moreover, by monitoring conversations regarding different domains of interests very useful insights were discovered. For our case, the most popular areas were:

\begin{tabular}{|c|c|c|}
\hline Name & Asociated & with \\
\hline Independence Anniversary & Post & weets \\
\hline State of the Nation Address & Post: & weets \\
\hline Development goals & Posts & weets \\
\hline Global poverty & Posts & weets \\
\hline National security & Posts, & weets \\
\hline Democracy & Posts, & weets \\
\hline Local development & Post & weets \\
\hline Bailout & Posts & weets \\
\hline Made In & Posts, & Wweets \\
\hline Public holidays & Post & Tweets \\
\hline NDC & Posts & Tweets \\
\hline Sports & Posts, & Tweets \\
\hline International security & Posts, & weets \\
\hline Public Administration & Posts & Tweets \\
\hline Telecommunication & Posts & weets \\
\hline Natural disasters & Posts & weets \\
\hline Criminal Justice System & Posts & weets \\
\hline Foreign affairs & Post & 2 Tweets \\
\hline National Development & Pos & Tweets \\
\hline Public events & Pos & Tweets \\
\hline Freedom of speech & Posts & weets \\
\hline Tourism & Posts & Tweets \\
\hline Ethnicity & Post: & weets \\
\hline Religion & Posts, & weets \\
\hline Mass media & Posts & weets \\
\hline Income & Post & weets \\
\hline Electricity & Posts, & Jweets \\
\hline
\end{tabular}

Table 2: Domain of interests (*sample data)

Besides posts' category, we realized that it is also important to understand what reaction generates each type of post: messages (text), videos, live videos, pictures or links:

Types of content that have highest engagement rate

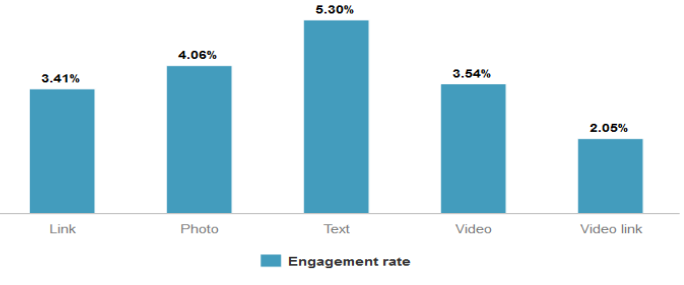

Figure 1: Content Type
Furthermore, subscribers' presence (tweets, likes/reactions, comments or shares) needs to be integrated in the analysis. We need to see how the post reached the subscriber: paid, organic or viral reach.

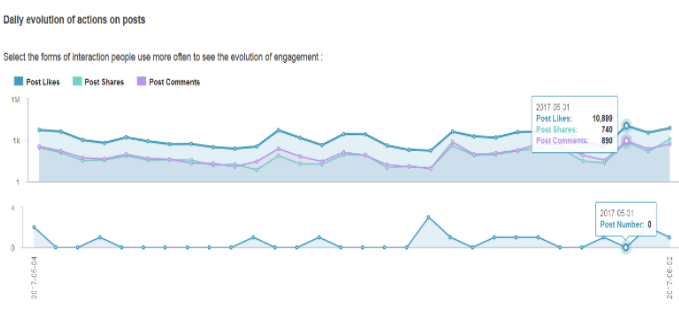

Figure 2: Daily evolution of actions on posts

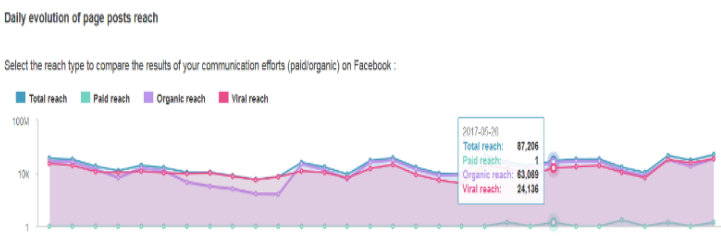

Figure 3: Daily evolution of posts reach

We understood that based on their reaction and presence, subscribers can have various levels of influence [Lazarsfeld and Merton et al 1954]. Therefore, it is important to identify the most 'vocal' followers and understand what are their beliefs. Based on the message that a politician wants to deliver, he can target distinct groups of people and use them to spread his ideas. In order to build a stronger engagement, we defined a gamification process as well.

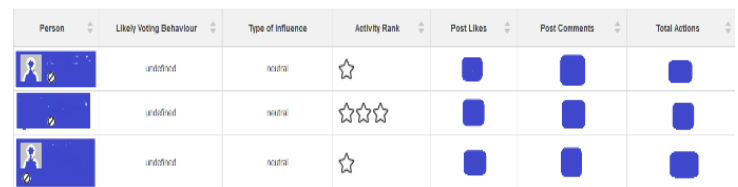

Figure 4: Level of influence

All the above indicators are enriched with statistics regarding the feedback received from subscribers to politician's posts (sentiment analysis): 

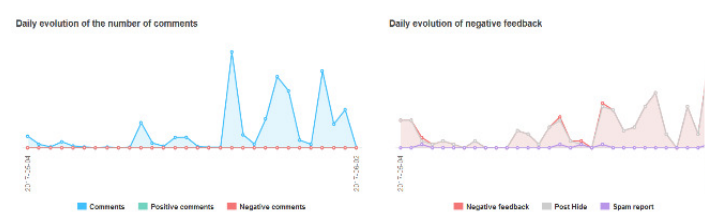

Figure 5: Evolution of positive / negative feedback
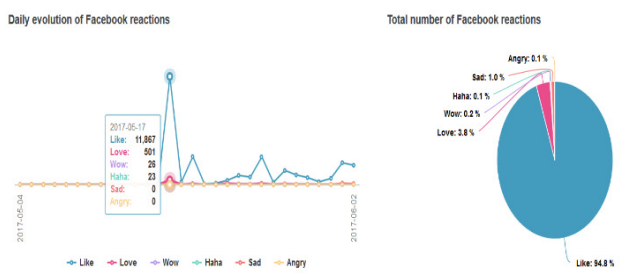

Figure 6: Reactions Evolution

In the end, everything is summarized in a single view:
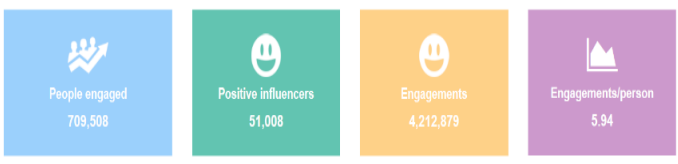

Figure 7: Summarization

In order to assess a politician communication strategy, it is essential to analyze the competition as well. Therefore, it is important to perform a competitive analysis - a comparison between the impact of a politician message versus his competitors.

Moreover, we analyze what were competitors' reactions to our candidate message (in case they had any reaction), what do we know about their subscribers and what are their evolutions on social media:

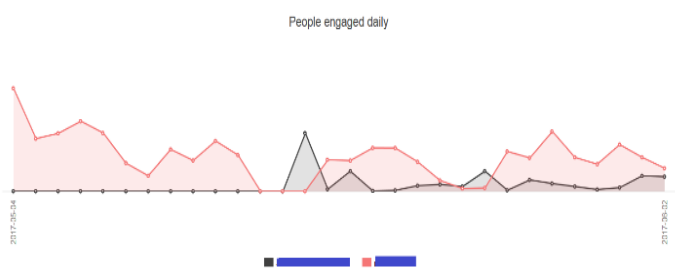

Figure 8: Competitive Analysis - Engagement Rate
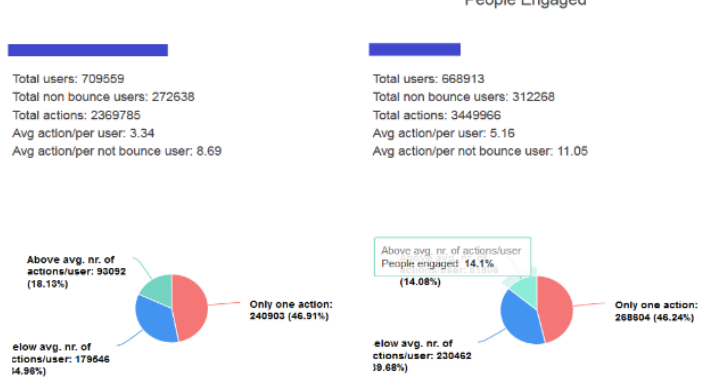

Figure 9: Competitive Analysis Metrics

The application can also perform competitive analysis between any two persons that have accounts on social media:

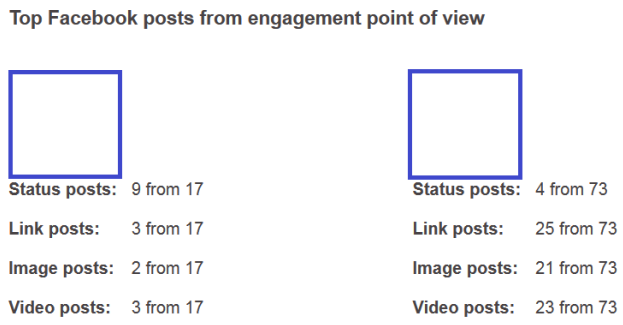

Figure 10: Facebook Competitive Analysis

\section{How it works}

Firstly, to obtain a proper overview about politician messages delivered on social media, it is essential to perform a strong analysis of the collected data: number of subscribers, number of likes, the reactions, shares, comments, etc.

The second step is data correlation and discovery. In this stage, we identify the main domain of interests by performing automatic classifications. We do a trend detection on topics, on message type, on subscribers' reaction. Also, we outline different typologies of subscribers' profiles and what are their level of influence.

The analysis is enriched with sentiment analysis performed using annotators. Majoritas Social Media Room profiles subscribers through the sentiment they associated with candidate's messages.

Moreover, we create multiple rankings calculated 
at a user level: activity rank, type of influence, social media influence rank, probable voting behavior.

In the end, we include competition analysis to get a complete overview regarding how our politician is placed in relation with his competitors.

By using all these functionalities, politicians can define their communication strategies. They can predict what type of feedback can be obtained by addressing different messages. Also, they can have a faster reaction and can apply agile methodology in defining communication plans.

\section{Conclusion and future work}

In this paper, we addressed the issue of getting political insights related to voters' reaction at politicians' message on social channels. We demonstrated that the political topic can influence the engagement rate and, the percentage of voters' favorability to a certain candidate.

Moreover, the authors observed a direct relationship between the number of supporters than a politician has on social media channels and the results of the elections. Also, we observed that the politicians that are active and react to users' comments are perceived as more favorable. This is trend is not applicable for CEE Europe, but for the all Europe and the US as well (Facebook followers: Hilary $10 \mathrm{~m}$ vs Trump $22 \mathrm{~m}$; Macron $1.9 \mathrm{~m}$ vs Le Pen $1.5 \mathrm{~m}$ ).

Majoritas Social Media Room was designed as end-to-end solution that assist politicians in calibrating their discourse and offer them the chance to customize their campaign based on people reaction.

Fortunately, there are no other similar platforms that perform this type of integrated assessment.

The automation of this creative process might be used not only in politics, but also in journalism or advertising.

As future work, we plan to enrich our platform with two new modules: one area regarding the prediction on the outcome of political events (there is a $\mathrm{x} \%$ chance that $\mathrm{Y}$ event is going to happen). Another area which can be improved is related to the surveys' assessment: the usage of the wisdom of crowds that perform much better than the media savvy pundits.

\section{References}

[Chilton and Schäffner et al 2002], Paul Chilton, Christina Schäffner in Politics as Text and Talk: Analytic approaches to political discourse, 2002

[Lazarsfeld and Merton et al 1954], Lazarsfeld P.F., Merton R.K. Friendship as a social process: a substantive and methodological analysis, 1954

[Liu et al 2012], Liu B. Sentiment Analysis and Opinion Mining. San Rafael, CA: Morgan \& Claypool; 2012

[Mullen and Malouf et al 2006], Mullen, T. and Malouf, R., AAAI: A Preliminary Investigation into Sentiment Analysis of Informal Political Discourse, 2006

[Phililips and Young et al 2009], Phillips D., \& Young, P., Online Public Relations: A Practical Guide to Developing an Online Strategy in the World of Social Media. London: Kogan Page; 2009

[Popescu and Etzioni et al 2005], Popescu, A.-M. \& Etzioni, O., Extracting Product Features and Opinions from Reviews. Proceedings of Conference on Empirical Methods in Natural Language; 2005

[Pozzi, Fersini, Mesina and Liu et al 2017], Pozzi F.A., Fersini E., Messina E. and Liu B., Sentiment Analysis in Social Networks, Elsevier First Edition, 2017

[Sundar, Kalyanaraman and Brwon et al 2003], Sundar, S. S., Kalyanaraman, S., \& Brown, J., Explicating Web Site interactivity: impression formation effects in Political Campaign Sites. Communication Research; 2003

[Tenhunen and Karvelyte et al 2015], Susanna Tenhunen and Vilma Karvelyte, European Parlamentary research Service Blog: The role played by social media in political participation and electoral campaigns, 2015

[Yu and Hatzivassiloglou et al 2003], Yu, H., and Hatzivassiloglou, V.,Towards answering opinion questions: Separating facts from opinions and identifying the polarity of opinion sentences, 2003 